\title{
Persistent sciatic vein
}

 \\ Department of Anatomy, Faculty of Medicine, University of Health Sciences, Istanbul, Turkey
}

[Received: 10 July 2021; Accepted: 5 September 2021; Early publication date: 15 September 2021]

\begin{abstract}
Background: Venous anomalies of the lower extremity are a challenge when found as rare variations. Physicians should be aware of morphological variations in this region for correct diagnosis and management of diseases such as deep vein thrombosis.

Materials and methods: During the routine dissection of the lower extremities of a Caucasian male cadaver, a case of persistent sciatic vein was found.

Results: A persistent sciatic vein originating from the saphenopopliteal junction coursed proximally and laterally to enter into the adductor magnus muscle, at the right side. Running through the fibres of the adductor magnus anteriorly, the variant vein drained into the deep femoral vein just medial to the vastus medialis muscle.

Conclusions: As being a rare congenital vascular anomaly, the persistent sciatic vein should be correctly diagnosed and defined during the radiologic assessments, surgical interventions and blockades for better outcomes and lesser complications. (Folia Morphol 2022; 81, 4: 1062-1065)
\end{abstract}

Key words: sciatic vein, popliteal vein, variation, cadaver, deep femoral vein, Klippel-Trénaunay-Weber syndrome

\section{INTRODUCTION}

During the $9^{\text {th }}$ to $15^{\text {th }}$ weeks of embryonic development of foetus, the large primitive axial vein drains the lower extremity. When the femoral vein grows into the main trunk of the thigh, the greater axial vein turns into a lesser arcade along the sciatic nerve. Persistence of this axial vein, which normally should regress, is defined as the persistent sciatic vein (PSV) [15]

Persistent sciatic vein, first described by Servelle in 1978 , is a rare congenital vascular anomaly characterised by varicose veins, cutaneous haemangiomas, and soft/hard tissue hypertrophy and it is often associated with Klippel-Trenaunay syndrome (KTS). The main venous outflow may be provided by a PSV when the femoral vein is occluded [3].
Classical textbooks define the popliteal vein (PV) to be formed by the junction of the anterior and posterior tibial veins at the lower border of the popliteus muscle. Crossing the popliteal fossa vertically, at its superior border, the vein passes through the adductor hiatus to become the femoral vein. Within the popliteal fossa, from superficial to deep, first the tibial nerve then the PV is encountered. The popliteal artery is located deep to the PV and it is in contact with the femur and the joint capsule. The PV and popliteal artery are in the same fibrous sheath. At the distal border of the popliteus the PV is posteromedial to the popliteal artery and superiorly it lies posterior to the artery $[7,12]$.

Both the anatomy and surgery books lack adequate descriptions of PV formation and its possible

Address for correspondence: H.A. Balcıoğlu, DDS, PhD, Department of Anatomy, Faculty of Medicine, University of Health Sciences, Üsküdar 38000 istanbul, Turkey, tel: +90 53052629 36, e-mail: habalci@gmail.com

This article is available in open access under Creative Common Attribution-Non-Commercial-No Derivatives 4.0 International (CC BY-NC-ND 4.0) license, allowing to download articles and share them with others as long as they credit the authors and the publisher, but without permission to change them in any way or use them commercially. 


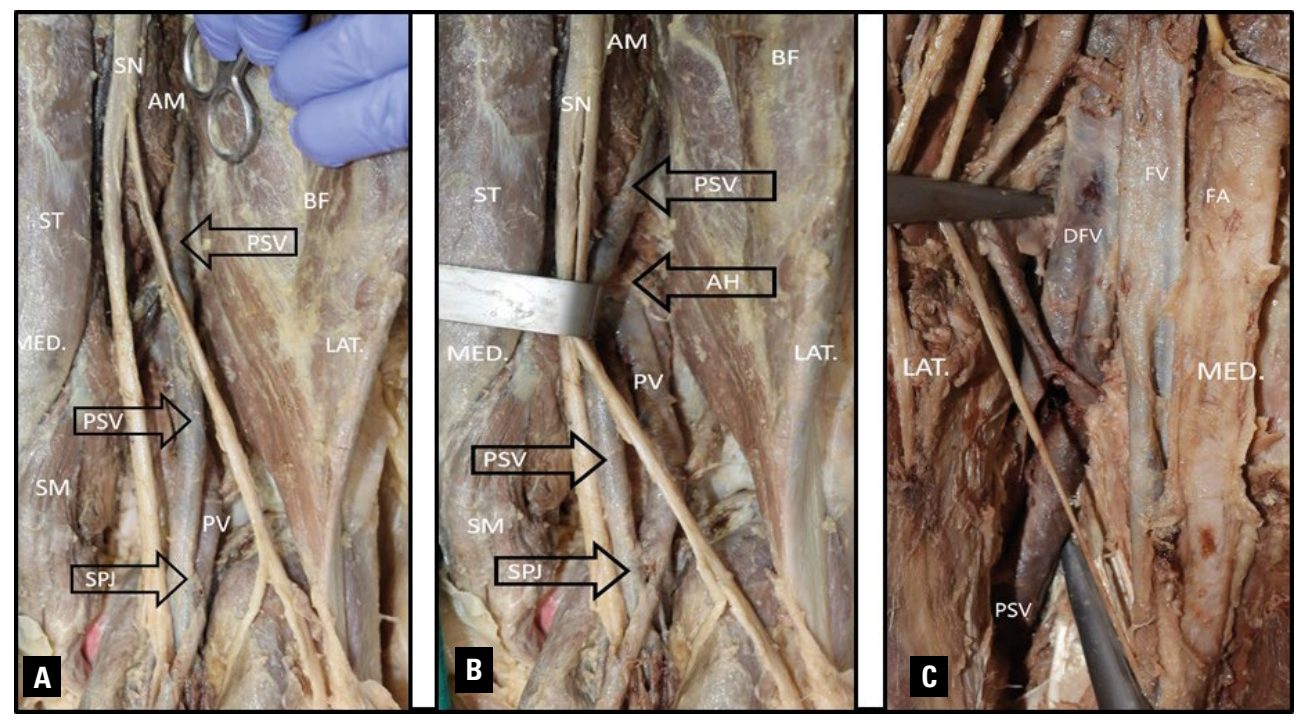

Figure 1. A. Posterior aspect of the right thigh: After removal of skin and superficial fascia biceps femoris retracted (with hand and scissor) to display the popliteal sciatic vein and adductor magnus; SN — sciatic nerve; AM — adductor magnus; ST — semitendinosus; BF — biceps femoris; PSV — popliteal sciatic vein; SM — semimembranosus; PV — popliteal vein; SPJ — saphenopopliteal junction; B. Posterior aspect of the right thigh with popliteal sciatic vein retracted: The photograph reveals the neurovascular structures of the right popliteal fossa and the penetration of the popliteal vein through the adductor hiatus. The retractor indicates the medial aspect of the thigh; AM — adductor magnus; BF — biceps femoris; SN — sciatic nerve; ST — semitendinosus; PSV — popliteal sciatic vein; AH — adductor hiatus; PV — popliteal vein; SM — semimembranosus; SPJ — saphenopopliteal junction; C. Anterior aspect of the right thigh: The photograph displays the drainage of the popliteal sciatic vein into the deep femoral vein on the anterior aspect of the right thigh. Deep femoral vein is located lateral to femoral vein. The top scissor indicates the lateral aspect of the thigh; FV — femoral vein; FA — femoral artery; DFV — deep femoral vein; PSV — popliteal sciatic vein.

variational patterns and also the multiple variational tributaries. Therefore, any case or research study might add to the comprehensive understanding of the nature of these vessels (Fig. 1A-C).

\section{CASE REPORT}

During the routine dissection of the lower extremities of a Caucasian male cadaver, a case of PSV was encountered at the right popliteal fossa. The variant vein originated from the saphenopopliteal junction (SPJ). While the PV was ascending anteriorly and medially to enter into the adductor canal, the variant vein was ascending laterally and penetrated the fibres of the adductor magnus muscle. The length of the variant vein, from the SPJ until its penetration into the adductor magnus muscle, was $15.17 \mathrm{~cm}$. The diameter of the vein at the emerging site was $8.58 \mathrm{~mm}$ and $8.61 \mathrm{~mm}$. The PV was $9.28 \mathrm{~mm}$ at the SPJ and $9.60 \mathrm{~mm}$ at the inferior hiatus of the adductor canal. A vein considerable in diameter $(3.85 \mathrm{~mm})$ draining the adductor magnus muscle opening into the PSV was observed. When the dissection was proceeded at the anterior surface of the thigh, the PSV was seen draining into the deep femoral vein just medial to the vastus medialis muscle. Small saphenous vein opened into the SPJ at its posterior aspect and the gastrocnemius vein at its medial aspect.

\section{DISCUSSION AND CONCLUSIONS}

Persistent sciatic vein may act as the main venous outflow tract in case femoral vein is occluded, though it may persist in the presence of patent superficial and deep femoral veins [1, 3]. The PSV in the present case with a diameter of $8 \mathrm{~mm}$ was observed together with a PV of a considerable size, which is $9 \mathrm{~mm}$ in diameter; that means the drainage of the leg was shared by these two veins.

Klippel-Trénaunay-Weber syndrome (KTWS) displays hypoplasia or aplasia of the femoral vein as a major finding together with the persistent sciatic vein, since the PSV serves as a compensatory venous pathway [6]. Peirce and Funaki reported the greater saphenous vein, deep femoral veins, and distal superficial femoral vein to be absent in a case of PSV in a patient with KTWS [9]. All of these veins were morphologically normal in our case. According to Servelle, the pathophysiology of KTWS is associated with the malformation of the PV. In this condition the venous drainage is maintained around the knee by the development of collaterals. One of these collaterals is 
PSV. However, the PV was evaluated and there was not a sign of occlusion or contraction in the present case. PSV is not unique to KTS; Cherry et al. [3] found only 20 patients with PSV in 279 patients with KTWS [11].

Several reports are found implicating the popliteal venous ligation as a contributing factor to the lower extremity amputations due to the fractures, gun wounds, blunt and/or penetrating trauma of the lower extremity [4]. Though the existence of variational veins such as PSV can be considered as an advantage in such conditions, because ligation of PV or PSV can be compensated by other vein, consequently it is important for the surgeons to consider the variational anatomy of the region very well before surgery.

This embryonic vascular remnant may be found in three morphological forms. The PSV is considered to be "complete" if courses along the thigh and buttock. A complete PSV arises from the PV, ascends to traverse the sciatic notch, and terminates draining into the internal iliac vein. An "upper" PSV arises from the minor tributaries of the upper thigh and passes through the sciatic notch ending in the pelvis. A "lower" PSV is limited to the distal and middle thigh opening into the deep femoral veins. Lower PSVs may also drain into an embryonic subcutaneous venous plexus [3]. Considering these patterns, the PSV in our case was a typical "lower" one draining into the deep femoral vein.

Cross et al. [4] investigated the vessels in the popliteal fossa of 52 lower extremities and 63 venograms and categorised the PVs in nine groups, but they did not mention PVS in any way. They described the origin of the PV occurring as the junction of medial and lateral veins. Out of nine groups, one group fits our case, whose prevalence they estimated at $1.9 \%$. According to the drawings of them, medial vein was the continuation of the posterior tibial vein and lateral vein was the continuation of the fibular vein, but the terminations of these two branches were not detailed [4].

Koç et al. [5] reported a bilateral PSV case in which both PVs were hypoplastic, contrary to our case. They reported the diameter of $\mathrm{PV}$ at the right side to be $5.21 \mathrm{~mm}$, while we measured the diameter of $\mathrm{PV}$ as $9.3 \mathrm{~mm}$ in our case. In their case, the origin of the PSV was anterior tibial vein at the right side and junction of the anterior and posterior veins at the left side. In our case PSV originated from the SPJ. Koç et al. [5] reported the diameters of the PSVs at the origin and termination to be $7.08 \mathrm{~mm}$ and 8.53 $\mathrm{mm}$, respectively at the right side while $7.78 \mathrm{~mm}$ and $7.43 \mathrm{~mm}$ at the left side. Ours were 8.6 and $9.6 \mathrm{~mm}$, respectively. The PSVs in both case reports drained into the deep femoral vein [5]. In our case, there was not an accompanying artery at the back of the thigh and the variant vein had no communication with the inferior gluteal vein or perforating veins along its course.

A solid knowledge of the variational venous anatomy of the lower extremities is crucial for the accurately assessment of the computed tomography scans and ultrasound examinations. Being aware of the diverse femoral and PV variations may be helpful to comprehensively interpret the suspected deep vein thrombosis and plan the interventional management [8]. Trigaux et al. [13] determined the presence of the sciatic venous axis and the possible incompetency of the deep venous system, stressing the clinical consequences such as the rejection of the saphenectomy. Such variant veins are regarded as a risk of complication during popliteal sciatic nerve blockade [5]. To avoid this kind of complications ultrasound visualisation of the region can help or, more classically, an aspiration may prevent an intravascular injection.

During the sciatic nerve decompression, the transgluteal approach is the conventional route through which the piriformis is reached and transected. Tubbs et al. [14] defined a new method for this procedure reaching the piriformis via the obturator canal in prone position. A potential presence of the PSV is very important, as in our case, since this new procedure is performed through adductor muscles. On the other hand, a complete PSV might be an obstacle during the transgluteal approach [14].

When a literature search on the PSV is performed, a few studies or case reports conducted by ultrasound and venography, poorly defining the origin, course, and termination of this congenital angiodysplasia, are found. An incidence of $0.7 \%$ to $9 \%$ appears as a result of overall review $[8,13]$. Cardoso et al. [2], after analysing 32 lower limbs of cadavers, reported the incidence of PSV as $6.25 \%$. Park et al. [8] detected 3 cases of PSV out of 445 patients in a retrospective computed tomography venography research. In their research conducted on 41 cadavers and 169 phlebographies, Pompeo et al. [10] reported the incidence as $3.33 \%$. Trigaux et al. [13] reported 7 patients with sciatic drainage pathway in more than 1200 ascending phlebographies in which no incidence is implicated. 
On the other hand, there stands a question if the incidences claimed in the aforementioned studies are reliable since magnetic resonance angiography is the most suitable imaging technique to make a certain diagnosis [1] and solely the cadaveric dissections could reveal the exact emergence, course and termination of the variant vessels.

\section{Conflict of interest: None declared}

\section{REFERENCES}

1. Bastarrika G, Redondo P. Indirect MR venography for evaluation and therapy planning of patients with Klippel-Trenaunay syndrome. Am J Roentgenol. 2010; 194(2): W244-W245, doi: 10.2214/AJR.09.3417, indexed in Pubmed: 20093583.

2. Cardoso $B$, Alvarenga $C$, Miyahara $M$, et al. Persistência da veia ciática. J Vasc Brasileiro. 2010; 9(3): 137-140, doi: 10.1590/s1677-54492010000300007.

3. Cherry K, Gloviczki P, Stanson A. Persistent sciatic vein: diagnosis and treatment of a rare condition. J Vasc Surg. 1996; 23(3): 490-497, doi: 10.1016/s07415214(96)80016-4.

4. Cross L, Hall J, Howdieshell T, et al. Clinical anatomy of the popliteal blood vessels. Clin Anat. 2000; 13(5): 347-353, doi: 10.1002/1098-2353(2000)13:5<347::aid-ca4>3.0.co;2-9.

5. Koç T, Gilan IY, Külekçi GD, et al. Bilateral persistent sciatic vein: report of a case with developmental, histological and clinical aspects. Surg Radiol Anat. 2014; 36(2): 189-194, doi: 10.1007/s00276-013-1146-9, indexed in Pubmed: 23748841.

6. Mavili E, Ozturk M, Akcali Y, et al. Direct $C T$ venography for evaluation of the lower extremity venous anomalies of Klippel-Trenaunay Syndrome. Am J Roentgenol. 2009; 192(6): W311-W316, doi: 10.2214/AJR.08.1151, indexed in Pubmed: 19457795.
7. Moore KL, Dalley AF. Clinically oriented anatomy. Wolters Kluwer, India Pvt Ltd 2018.

8. Park EA, Chung JW, Lee W, et al. Three-dimensional evaluation of the anatomic variations of the femoral vein and popliteal vein in relation to the accompanying artery by using CT venography. Korean J Radiol. 2011; 12(3): 327-340, doi: 10.3348/kjr.2011.12.3.327, indexed in Pubmed: 21603292.

9. Peirce RM, Funaki B. Direct MR venography of persistent sciatic vein in a patient with Klippel-Trenaunay-Weber syndrome. Am J Roentgenol. 2002; 178(2): 513-514, doi: 10.2214/ajr.178.2.1780513, indexed in Pubmed: 11804934.

10. Pompeo A, Grinberg $H$, Burihan $M$, et al. Quão infrequente é a veia isquiática. Anais do Congresso Brasileiro de Angiologia e Cirurgia Vascular publicado no. J Vasc Brasileiro. 2003; 2: 129.

11. Servelle M. Klippel and Trénaunay's syndrome. 768 operated cases. Ann Surg. 1985; 201(3): 365-373, doi: 10.1097/00000658-198503000-00020, indexed in Pubmed: 2983626.

12. Standring S. Gray's Anatomy. The Anatomical Basis of Clinical Practice. 41th ed. Standring S (ed). Elsevier, 25th September 2015.

13. Trigaux JP, Vanbeers BE, Delchambre FE, et al. Sciatic venous drainage demonstrated by varicography in patients with a patent deep venous system. Cardiovasc Intervent Radiol. 1989; 12(2): 103-106, doi: 10.1007/BF02577400, indexed in Pubmed: 2500240.

14. Tubbs RI, Barton JC, Watson CC, et al. et al., et al.. A novel method for sciatic nerve decompression: Cadaveric feasibility study with potential application to patients with piriformis syndrome. Transl Res Anat. 2015; 1: 40-43, doi: 10.1016/j.tria.2015.10.006.

15. Uhl JF. Uhl J-F. Focus on venous embryogenesis of the human lower limbs. Phlebolymphology. 2015; 22: $55-63$. 